\title{
Introduction to The Origin of Electromagnetic Mass (Electromagnetic Inertia)
}

$\underline{\text { Wim Vegt }}$

\section{Eindhoven University of Technology}

\section{Inertia and the change in the relative radiation pressure}

To understand this mathematical approach in physics, it is necessary to understand the concept of "inertia". The property of inertia for "matter" as well the property of inertia for "light".

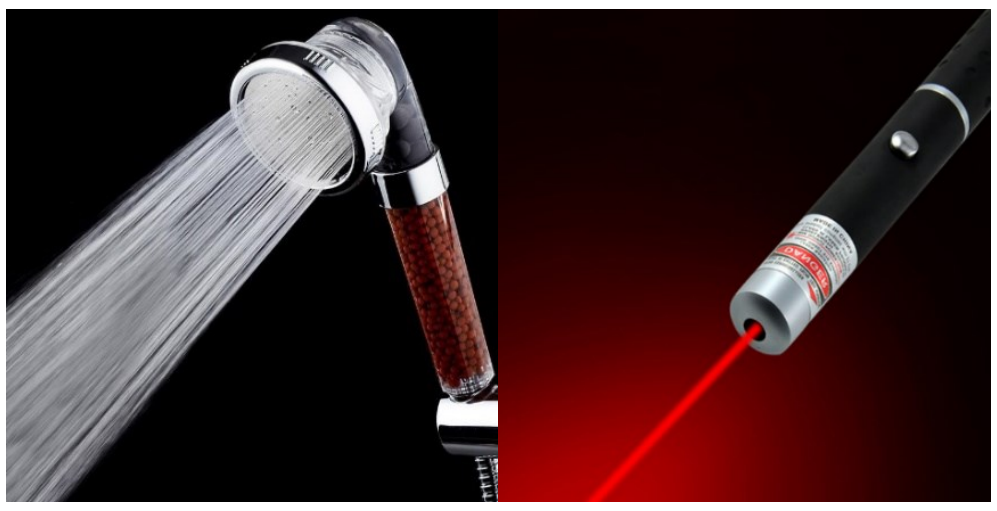

Figure 1 The comparison of a waterjet emitted by a shower head and a laser beam emitted by a laser.

To understand the property of "inertia" for a beam light, we start with an imaginary model of a beam of light, presented by a jet of water leaving a shower head.

Imagine you hold your right hand in the water jet, emitted by a shower head. Then you will feel the radiation pressure of the water jet, comparable with the radiation pressure of a beam of light emitted by a laser.

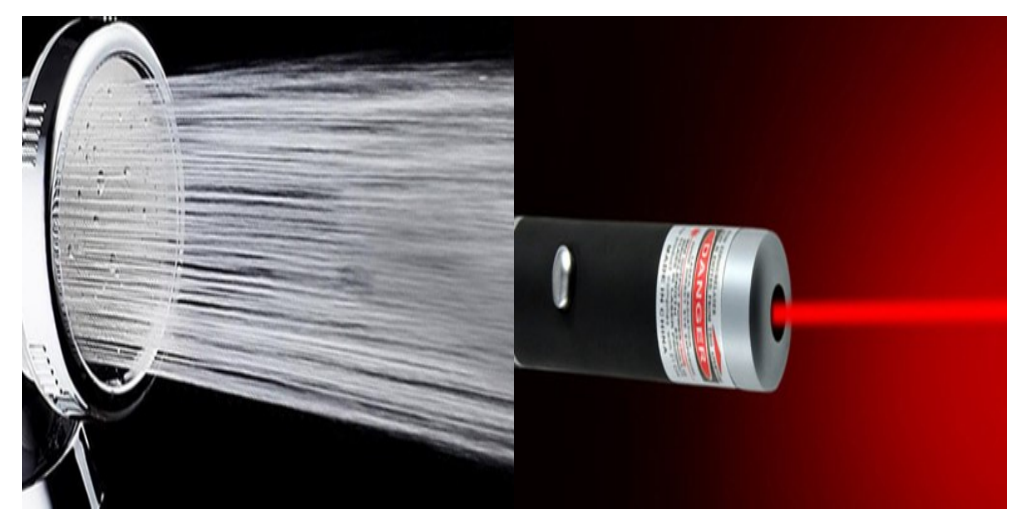

Figure 2 The shower head emits a waterjet towards to the right and the laser emits a laser beam towards to the right.

When you move your right hand towards to the left (in the opposite direction of the water jet), you will feel that the radiation pressure of the water jet increases. When you move your 
hand towards to the right (in the direction of the water jet) you will feel that the radiation pressure of the water jet decreases.

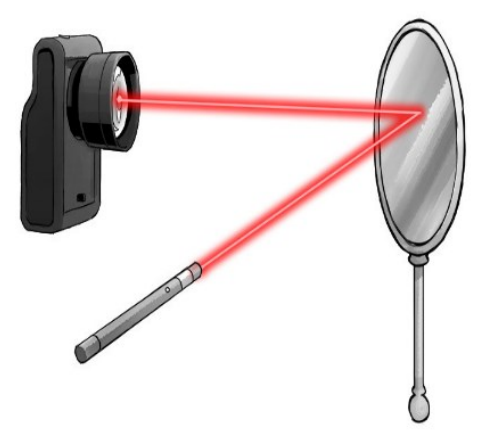

Figure 3. A laser beam has been reflected by the mirror (placed at the right from the laser) and acts with an electromagnetic radiation pressure on the mirror directed towards to the right.

In a comparable way the radiation pressure of a laser beam emitted by a laser acting on the mirror, will increase when we move the mirror towards to the left (towards the laser, in the opposite direction of propagation of the laser beam). The radiation pressure on the mirror will decrease when we move the mirror towards to the right (away from the laser, in the direction of propagation of the laser beam).

Of course, the radiation pressure of the beam of laser light will be much smaller compared to the radiation pressure of the water jet. But with very sensitive equipment the radiation pressure of the beam of laser light can be measured. As an example, the radiation pressure on earth of the light emitted by the sun, equals about $10\left[\mu \mathrm{N} / \mathrm{m}^{2}\right]$. Which equals a total radiation pressure of the sunlight acting on the surface of the whole earth of about $1.2510^{9}[\mathrm{~N}]$.

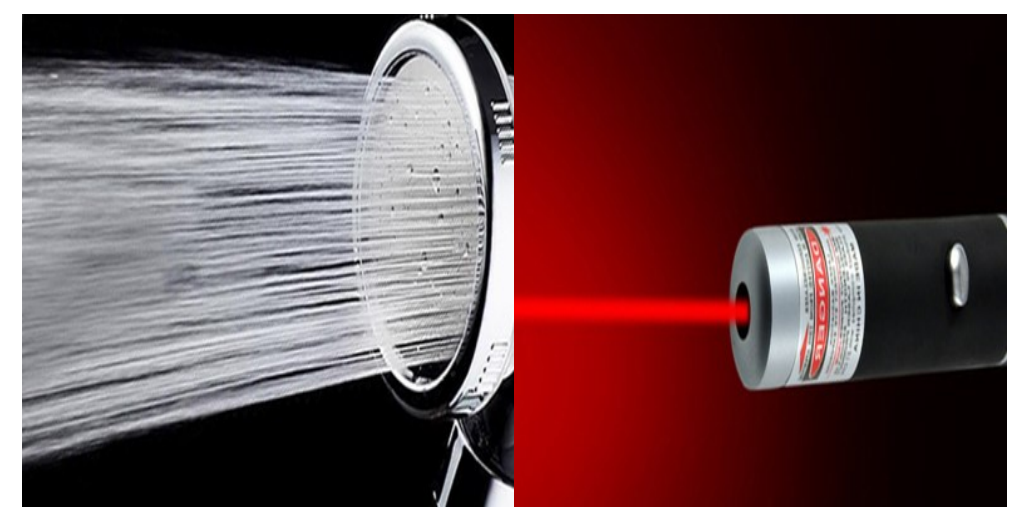

Figure 4. The shower head emits a waterjet towards to the left and the laser emits a laser beam towards to the left. 
Now we choose a shower head and a laser emitting towards the left. When you move your left hand towards to the left (in the direction of the water jet), you will feel that the radiation pressure of the water jet decreases. When you move your left hand towards to the right (in the opposite direction of the water jet) you will feel that the radiation pressure of the water jet increases.

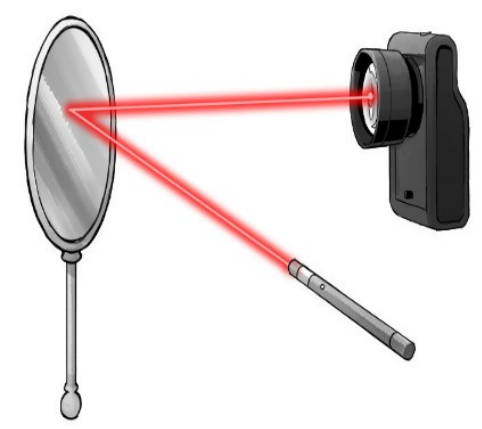

Figure 5. A laser beam has been reflected by the mirror (placed at the left from the laser) and acts with an electromagnetic radiation pressure on the mirror directed towards to the left.

In a comparable way the radiation pressure of a laser beam emitted by a laser acting on the mirror, will decrease when we move the mirror towards to the left (away from the laser, in the direction of propagation of the laser beam). The radiation pressure on the mirror will increase when we move the mirror towards to the right (in the opposite direction of the laser beam).

\subsection{Inertia and the relative change of the radiation pressures of confined water jets}

To demonstrate the property of inertia, we place the right hand in the water jet, emitted towards to the right by the first shower head. The the left hand has been placed in the water jet, emitted towards to the left by the second shower head.

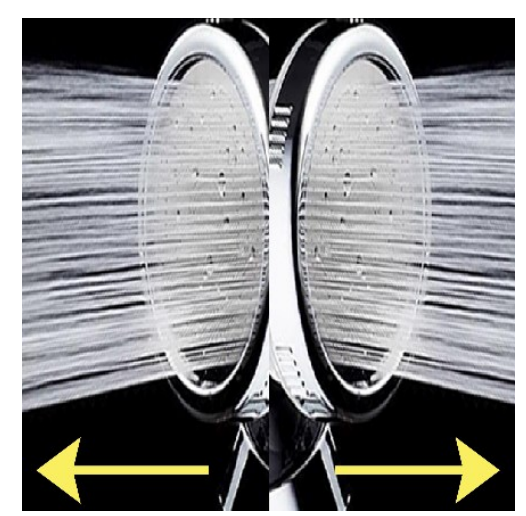

Figure 6. Two shower heads, pointing in opposite directions, emit two waterjets pointing in opposite directions. The left hand has been placed in the waterjet emitted towards to the left and the right hand has been placed in the waterjet emitted towards to the right.

The radiation pressure of the water jet acting on the left hand equals the radiation pressure acting on the right hand but has been directed oppositely. Both radiation pressures neutralize each-other and the resulting force acting on the system of both hands equals zero. 


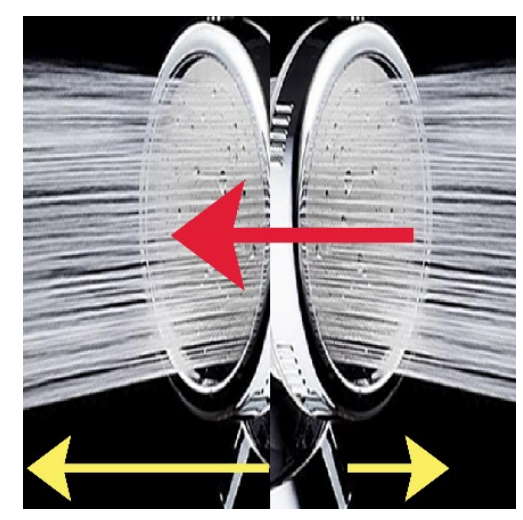

Figure 7. The left hand has been placed in the waterjet emitted towards to the left and the right hand has been placed in the waterjet emitted towards to the right. The resulting force has been oriented towards to the Left.

To demonstrate the property of inertia of the confined water jets between both hands, we move both hands simultaneously towards to the right. The radiation pressure of the waterjet acting on the left hand will increase. The radiation pressure of the waterjet acting on the right hand will decrease. The total resulting radiation pressures of both waterjets will not neutralize each-other anymore. The total resulting radiation pressure of both waterjets will be directed towards to the left, opposite in the direction of moving. In figure 7 this has been represented by the red arrow pointing towards to the left. We experience the effect of inertia. Both waterjets resist the starting movement of our both hands towards to the right, when we consider both hands together as one mechanical system.

We replace both hands by one (plastic) box, placed over both waterjets. When we move the box towards to the right, we will experience the (extra) inertia of the box, caused by the resulting force of both waterjets directed towards to the left. This is the resulting inertia force, represented by the red arrow in figure 7 . Moving the box towards to the right results in an inertia force in the opposite direction represented by the red arrow in figure 7.

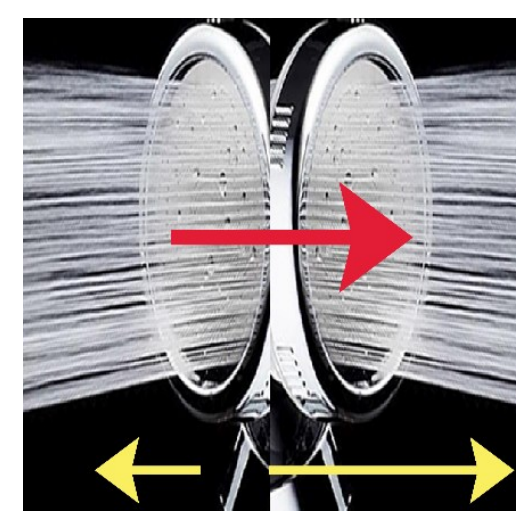

Figure 8. The left hand has been placed in the waterjet emitted towards to the left and the right hand has been placed in the waterjet emitted towards to the right. The resulting force has been oriented towards to the Right.

To demonstrate the property of inertia of the confined water jets between both hands, we move both hands simultaneously towards to the left. The radiation pressure of the waterjet acting on the left hand will decrease. The radiation pressure of the waterjet acting on the right hand will decrease. The total resulting radiation pressures of both waterjets will not neutralize 
each-other anymore. The total resulting radiation pressure of both waterjets will be directed towards to the left, opposite in the direction of moving. In figure 7 this has been represented by the red arrow pointing towards to the left. We experience the effect of inertia. Both waterjets resist the starting movement of our both hands towards to the left, when we consider both hands together as one mechanical system.

We replace both hands by one (plastic) box, placed over both waterjets. When we move the box towards to the right, we will experience the (extra) inertia of the box, caused by the resulting force of both waterjets directed towards to the left. This is the resulting inertia force, represented by the red arrow in figure 8 . Moving the box towards to the left results in an inertia force in the opposite direction represented by the red arrow in figure 8 .

\subsection{Inertia and the relative change of the radiation pressures of confined laser beams}

To demonstrate the property of inertia of the electromagnetic radiation (laser beam), confined between both mirrors, we place the right mirror in the laser beam, emitted towards to the right by the first laser. The left mirror has been placed in the laser beam, emitted towards to the left by the second laser.

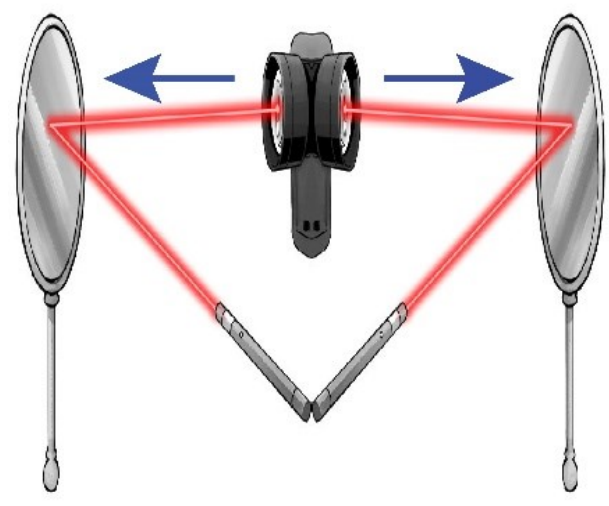

Figure 9. Two lasers, pointing in opposite directions, emit two laser beams pointing in opposite directions. The left mirror has been placed in the laser beam emitted towards to the left and the right mirror been placed in the laser beam towards to the right

The radiation pressure of the laser beam acting on the left mirror equals the radiation pressure acting on the right mirror but has been directed oppositely. Both radiation pressures neutralize each other, and the resulting force acting on the system of both mirrors equals zero.

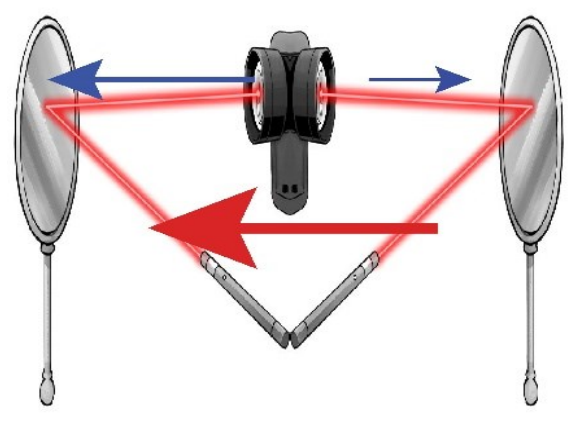


Figure 10. The left mirror has been placed in the laser beam emitted towards to the left and the right mirror been placed in the laser beam towards to the right. The resulting force has been directed towards tot the Left.

To demonstrate the property of inertia of the confined laser beams between between both mirrors, we move both mirrors simultaneously towards to the right. The radiation pressure of the laser beam acting on the left mirror will increase. The radiation pressure of the laser beam acting on the right mirror will decrease. The total resulting radiation pressures of both laser beams will not neutralize each-other anymore. The total resulting radiation pressure of both laser beams will be directed towards to the left, opposite in the direction of moving. In figure 10 this has been represented by the red arrow pointing towards to the left. We experience the effect of inertia. Both laser beams resist the starting movement of our both mirrors, when we consider both mirrors together as one mechanical system.

We replace both mirrors by a box with at the inside $100 \%$ reflecting mirrors. The confined electromagnetic radiation (laser beam) between both mirrors will act on both mirrors with an oppositely directed radiation pressure.

When we start to move the box towards to the right, the resulting total radiation pressure will be oriented towards tot the left. This is the inertia force of the confined electromagnetic radiation. Albert Einstein demonstrated the proportionality between mass (inertia) and energy (intensity of electromagnetic radiation of the confined laser beam) by his famous equation: $\mathrm{E}$ $=\mathrm{m} \mathrm{c}^{2}$.

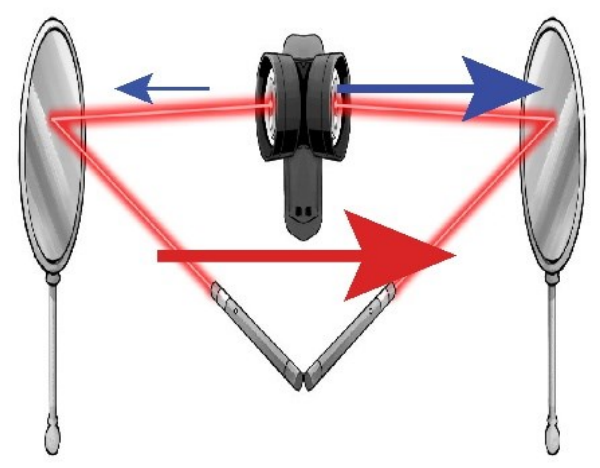

Figure 11. The left mirror has been placed in the laser beam emitted towards to the left and the right mirror been placed in the laser beam towards to the right. The resulting force has been directed towards tot the Right.

To demonstrate the property of inertia of the confined laser beams between between both mirrors, we move both mirrors simultaneously towards to the left. The radiation pressure of the laser beam acting on the left mirror will decrease. The radiation pressure of the laser beam acting on the right mirror will increase. The total resulting radiation pressures of both laser beams will not neutralize each-other anymore. The total resulting radiation pressure of both laser beams will be directed towards to the right, opposite in the direction of moving. In figure 11 this has been represented by the red arrow pointing towards to the right. We experience the effect of inertia. Both laser beams resist the starting movement of our both mirrors, when we consider both mirrors together as one mechanical system. 
We replace both mirrors by a box with at the inside $100 \%$ reflecting mirrors. The confined electromagnetic radiation (laser beam) between both mirrors will act on both mirrors with an oppositely directed radiation pressure.

When we start to move the box towards to the left, the resulting total radiation pressure will be oriented towards tot the right. This is the inertia force of the confined electromagnetic radiation. Albert Einstein demonstrated the proportionality between mass (inertia) and energy (intensity of electromagnetic radiation of the confined laser beam) by his famous equation: $\mathrm{E}$ $=\mathrm{m} \mathrm{c}^{2}$.

We replace in a "thought experiment" the box with at the inside $100 \%$ reflecting mirrors by a sphere with a $100 \%$ reflecting inside. The hollow sphere contains confined electromagnetic radiation. Proportional to the total confined electromagnetic energy inside the sphere, the sphere will demonstrate the property of "inertia" in any direction according Newton's second law of motion: $\mathrm{F}=\mathrm{m}$. a.

Now we replace the hollow sphere with confined electromagnetic radiation by a "Gravitational-Electromagnetic Confinement" of electromagnetic radiation. In a comparable way the electromagnetic confinement will demonstrate the property of "inertia", represented by Newton's second law of motion: $\mathrm{F}=\mathrm{m}$. a.

\subsection{The anisotropic effects of inertia}

A laser beam demonstrates an "anisotropic" effect of inertia. Which means that the effect of inertia is not the same in any direction, like the inertia of confined electromagnetic radiation within a hollow sphere. Only "Confined Electromagnetic Energy" demonstrates the effect of inertia according Newton's second law of motion.

In the directions, perpendicular to the direction of propagation of the laser beam, the electromagnetic energy of the Laser beam has been confined. In these directions, perpendicular to the direction of propagation, the laser beam acts according Newton's second law of motion $\mathrm{F}=\mathrm{m}$. a. The mass density of the laser beam has been determined by Einstein's $\mathrm{E}=\mathrm{m} \mathrm{c}^{2}$. When we divide the left and right terms of Einstein's equation by the Volume, we find a relationship between the energy density " $w$ " and the mass density " $\rho$ ". Einstein's equation can be written as: $\mathrm{w}=\rho \mathrm{c}^{2}$.

This equation is the fundamental equation for the representation of a "Transversal Black Hole" in which a beam of light follows a circular orbit around a Black Hole due to the Gravity force acting on the mass density " $\rho$ " of the beam of light. Comparable with a satellite moving in a (circular) orbit around the earth because of the gravity force of the earth acting on the mass of the satellite.

In the direction of propagation, the electromagnetic energy has not been confined. In the direction of the propagation of a laser beam does exist a perfect equilibrium between the forward directed radiation pressure and the inertia (mass) of the electromagnetic energy density " $\rho$ ". For this reason, it is impossible to accelerate or to decelerate the propagation speed of a laser beam according Newton's second law of motion.

This is comparable with the speed of sound for a sound wave, which will not be influenced by the existence of a gravitational field. A beam of light moving towards a Black Hole or 
away from a Black Hole will keep the same speed of light. But the intensity of the beam of Light will increase when the beam of light moves towards a Black Hole and the intensity will decrease when a beam of light moves away from a Black Hole. This type of confinement has been called a "Longitudinal Black Hole" in which the confined light always propagates in the direction of the gravitational field of the Black Hole.

\section{Inertia}

Newton described in his second law of motion the classical definition of mass (inertia). However, it is impossible to calculate with Newton's second law of motion the (electromagnetic) mass of a beam of light (Ref. [1], [2],[3]). Because the speed of light is a universal constant which follows from Albert Einstein's Theory of Special Relativity, it is impossible to accelerate or to slow down a beam of light and for that reason it is impossible to determine the electromagnetic mass of a beam of light (free electromagnetic radiation) by Newton's second law.

To calculate the electromagnetic mass of free or confined electromagnetic radiation, the fundamental concept of the New Theory has been used that the Universe is in a perfect Equilibrium and that any electromagnetic field configuration is in a perfect equilibrium with itself and its surrounding. From this fundamental concept follows a different definition of (confined) electromagnetic mass.

Electromagnetic mass (or inertia) has been determined by the relativistic Lorentz transformation of the radiation pressures in all different directions and the disturbance of a uniform motion (or position at rest) of confined electromagnetic radiation results in a relativistic effect which we measure (experience) as electromagnetic mass (inertia).

The mass in $[\mathrm{kg}]$ of an object will be generally measured by acceleration (or deceleration) of the object according Newton's second law of motion. In the theory of special relativity, the speed of light is a fundamental constant and the intensity of the light is not a universal constant.

The calculate the relativistic mass of Confined Electromagnetic Radiation, we start with a thought experiment in which a beam of light is propagating between two $100 \%$ reflecting mirrors, indicated as Mirror A and Mirror B. Both mirrors are part of a rigid construction and the relative velocity between both mirrors always equals zero. The results of this calculation will be generalized for any kind of electromagnetic radiation which has been confined by its own electromagnetic and gravitational field. When the speed of the observer has the same speed as the speed of the light source, then the observer and the light source are relative at rest. And the same light intensity will be measured at the location of the emitter and at the location of the observer. 


\section{Newton's first law for confined Electromagnetic Radiation.}

When the observer moves towards the emitter, the intensity of the light at the location of the observer will increase with $\gamma(1+\mathrm{v} / \mathrm{c})$ according the Lorentz transformation in which " $\mathrm{v}$ " is the relative velocity between emitter and observer. At low velocities the term $\gamma$ will equal 1 .

When the observer moves away from the emitter, the intensity of the light at the location of the observer will decrease with $\gamma(1-\mathrm{v} / \mathrm{c})$ according the Lorentz transformation. At low velocities the term:

$$
\gamma=\frac{1}{\sqrt{1-\frac{\mathrm{v}^{2}}{\mathrm{c}^{2}}}}
$$

will equal 1 ( $v$ equals the relative velocity between object and observer and c equals the speed of light).

When light is confined between two $100 \%$ reflecting mirrors, then we can conclude that the speed of both mirrors will always be equal, relative to each other. And at uniform speed, the radiation pressures on both mirrors will be equal and both opposite directed radiation pressures will neutralize.

\section{Newton's second law of motion for confined Electromagnetic Radiation.}

During acceleration, it will take time for the light to travel with the speed of light between both mirrors. When we consider one mirror as the emitter and the opposite mirror as the observer, we can conclude that the speed of the emitter will be different (when the beam of light leaves the emitter) than the speed of the observer (when the beam of light reaches the observer) because of the time interval, needed for the beam of light to propagate from emitter to observer during the acceleration. During the acceleration, both opposite oriented radiation pressures on both mirrors will not be equal anymore and they will not neutralize each other anymore. During acceleration, there will be a resulting force according Newton's second law of motion caused by both different radiation pressures.

For the first step in this calculation an imaginary experiment has been used. Two $100 \%$ reflecting mirrors $\mathrm{B}$ and $\mathrm{A}$ (both in the $\mathrm{x}-\mathrm{y}$ plane and without any mass) are placed opposite each other at a distance $\Delta \mathrm{x}$ [10]. A single harmonic electromagnetic wave has been confined between both mirrors. Between both mirrors a "Standing Electromagnetic Wave" appears which has been formed by two waves travelling in opposite directions along the z-axis.

The Poynting vector corresponding with the electromagnetic wave propagating along the $\mathrm{z}$ axis in the + direction (positive direction of the z-axis) has been indicated as $\overline{\mathrm{S}}^{+}=\overline{\mathrm{E}}^{+} \times \overline{\mathrm{H}}^{+}$and the Poynting vector corresponding with the electromagnetic wave propagating along the $\mathrm{z}$-axis in the - direction (opposite direction) has been indicated as $\bar{S}^{-}=\overline{\mathrm{E}}^{-} \times \overline{\mathrm{H}}^{-}$. The system is at rest. The radiation pressures, caused by the confined electromagnetic radiation, on both mirrors $\mathrm{A}$ 
and $\mathrm{B}$ are opposite and equal in magnitude:

$$
\mathbf{P}_{\mathrm{A}}=\frac{2 \mathrm{~S}_{\mathrm{A}}}{\mathrm{C}}=\frac{2 \mathrm{~S}_{\mathrm{B}}}{\mathrm{C}}=\mathbf{P}_{\mathrm{B}}
$$

Einstein has formulated this very well. "Velocities are always relative" . To calculate the radiation pressure on Mirror A, the velocities, only relative to Mirror A for the waves with the respective Poynting vectors $\overline{\mathrm{S}}^{+}=\overline{\mathrm{E}}^{+} \times \overline{\mathrm{H}}^{+}$and $\overline{\mathrm{S}}^{-}=\overline{\mathrm{E}}^{-} \times \overline{\mathrm{H}}^{-}$, have to be calculated.

\subsection{The radiation pressure on Mirror A, when Mirror A moves with a velocity $v$ in the direction of the positive z-axis:.}

When the system of "Two Mirrors B - A" moves in the direction of the positive z-axis, Mirror A moves in the direction of the positive z-axis and the Poynting vector $\bar{S}^{+}=\overline{\mathrm{E}}^{+} \times \overline{\mathrm{H}}^{+}$ will decrease according the Lorentz transformation [11].

$$
\bar{S}_{\mathrm{v}}^{+}=\overline{\mathrm{E}}_{\mathrm{v}}^{+} \times \overline{\mathrm{H}}_{\mathrm{v}}^{+}=\gamma^{2}\left(1-\frac{\mathrm{v}}{\mathrm{c}}\right)^{2}\left(\overline{\mathrm{E}}^{+} \times \overline{\mathrm{H}}^{+}\right)
$$

When the system of "Two Mirrors B - A" moves in the direction of the positive z-axis, Mirror A moves in the direction of the positive z-axis the Poynting vector $\bar{S}^{-}=\overline{\mathrm{E}}^{-} \times \overline{\mathrm{H}}^{-}$will increase according the Lorentz transformation [11].

$$
\bar{S}_{\mathrm{v}}^{-}=\overline{\mathbf{E}}_{\mathrm{v}}^{-} \times \overline{\mathbf{H}}_{\mathrm{v}}^{-}=\gamma^{2}\left(1+\frac{\mathrm{v}}{\mathrm{c}}\right)^{2}\left(\overline{\mathrm{E}}^{+} \times \overline{\mathbf{H}}^{+}\right)
$$

The total radiation pressure, caused by the confined electromagnetic radiation, on mirror A equals:

$$
\mathrm{P}_{\mathrm{A}}=\frac{\mathrm{S}_{A}^{+}+\mathrm{S}_{A}^{-}}{\mathrm{c}}=\frac{\gamma^{2}\left(\left(1-\frac{\mathrm{v}}{\mathrm{c}}\right)^{2}+\left(1+\frac{\mathrm{v}}{\mathrm{c}}\right)^{2}\right)\left(\overline{\mathrm{E}}^{+} \times \overline{\mathrm{H}}^{+}\right)}{\mathrm{c}}
$$




\subsection{The radiation pressure on Mirror $B$ when Mirror $B$ moves with a velocity $v$ in the direction of the positive z-axis:}

When the system of "Two Mirrors B - A" moves in the direction of the positive z-axis, Mirror B moves in the direction of the positive $\mathrm{z}$-axis and the Poynting vector $\bar{S}^{-}=\overline{\mathrm{E}}^{-} \times \overline{\mathrm{H}}^{-}$will increase according the Lorentz transformation [11].

$$
\overline{\boldsymbol{S}}_{\mathrm{v}}^{-}=\overline{\mathbf{E}}_{\mathrm{v}}^{-} \times \overline{\mathbf{H}}_{\mathrm{v}}^{-}=\gamma^{2}\left(1+\frac{\mathrm{v}}{\mathrm{c}}\right)^{2}\left(\overline{\mathbf{E}}^{+} \times \overline{\mathbf{H}}^{+}\right)
$$

When the system of "Two Mirrors B - A" moves in the direction of the positive z-axis, Mirror A moves in the direction of the positive z-axis the Poynting vector $\overline{\mathrm{S}}^{+}=\overline{\mathrm{E}}^{+} \times \overline{\mathrm{H}}^{+}$will increase according the Lorentz transformation [11].

$$
\bar{S}_{\mathrm{v}}^{+}=\overline{\mathrm{E}}_{\mathrm{v}}^{+} \times \overline{\mathrm{H}}_{\mathrm{v}}^{+}=\gamma^{2}\left(1-\frac{\mathrm{v}}{\mathrm{c}}\right)^{2}\left(\overline{\mathrm{E}}^{+} \times \overline{\mathrm{H}}^{+}\right)
$$

The total radiation pressure, caused by the confined electromagnetic radiation, on mirror $\mathrm{B}$ equals:

$\mathrm{P}_{\mathrm{A}}=\frac{\mathrm{S}_{A}^{+}+\mathrm{S}_{A}^{-}}{\mathrm{c}}=\frac{\gamma^{2}\left(\left(1+\frac{\mathrm{v}}{\mathrm{c}}\right)^{2}+\left(1-\frac{\mathrm{v}}{\mathrm{c}}\right)^{2}\right)\left(\overline{\mathrm{E}}^{+} \times \overline{\mathrm{H}}^{+}\right)}{\mathrm{c}}$

$P_{A}$ and $P_{B}$ are still equal in magnitude and both in opposite direction and still cancel each other. The system fulfils Newton's first law of motion. 


\subsection{Newton's second Law of Motion (Inertia) for Confined Electromagnetic Radiation:}

When the system of "Two Mirrors B - A" accelerates, the velocity increases with ${ }_{\Delta} \mathrm{v}$ in a time interval $\Delta \mathbf{t}$. At time $t$ the radiation pressures on mirror $A$ and mirror $B$ are presented in [10]) and [11]. At time $\mathbf{t}+\Delta_{\mathbf{t}}$ the radiation pressures on Mirror A and Mirror B will different:

The radiation pressure at time $\mathbf{t}+\Delta_{\mathbf{t}}$ caused by the confined electromagnetic radiation, on mirror A equals [11]:

$\mathrm{P}_{\mathrm{A}}=\frac{\mathrm{S}_{A}^{+}+\mathrm{S}_{A}^{-}}{\mathrm{c}}=\frac{\gamma^{2}\left(\left(1+\frac{(\mathrm{v})}{\mathrm{c}}\right)^{2}+\left(1-\frac{(\mathrm{v}+\Delta \mathrm{v})}{\mathrm{c}}\right)^{2}\right)\left(\overline{\mathrm{E}}^{+} \times \overline{\mathrm{H}}^{+}\right)}{\mathrm{c}}$

Because the wave with Poynting vector $\overline{\mathrm{S}}^{+}=\overline{\mathrm{E}}^{+} \times \overline{\mathrm{H}}^{+}$has left Mirror B at " $\mathrm{t}$ " and during the time interval $\Delta \mathrm{t}$ the magnitude of $\overline{\mathrm{E}}_{\mathrm{t}}^{+}=\left(1+\frac{\mathrm{v}}{\mathrm{c}}\right) \overline{\mathrm{E}}^{+}$and $\overline{\mathrm{H}}_{\mathrm{t}}^{+}=\left(1+\frac{\mathrm{v}}{\mathrm{c}}\right) \overline{\mathrm{H}}^{+}$has not changed.

The radiation pressure at time $\mathrm{t}+\Delta \mathrm{t}$ caused by the confined electromagnetic radiation, on mirror B equals:

$\mathrm{P}_{B}=\frac{\mathrm{S}_{B}^{+}+\mathrm{S}_{B}^{-}}{\mathrm{c}}=\frac{\gamma^{2}\left(\left(1+\frac{(\mathrm{v}+\Delta \mathrm{v})}{\mathrm{c}}\right)^{2}+\left(1-\frac{(\mathrm{v})}{\mathrm{c}}\right)^{2}\right)\left(\overline{\mathrm{E}}^{+} \times \overline{\mathrm{H}}^{+}\right)}{\mathrm{c}}$

Because the wave with Poynting vector $\bar{S}^{-}=\overline{\mathrm{E}}^{-} \times \overline{\mathrm{H}}^{-}$has left Mirror A at " $\mathrm{t}$ " and during the time interval $\Delta \mathrm{t}$ the magnitude of $\overline{\mathrm{E}}_{\mathrm{t}}^{-}=\left(1+\frac{\mathrm{v}}{\mathrm{c}}\right) \overline{\mathrm{E}}^{-}$and $\overline{\mathrm{H}}_{\mathrm{t}}^{-}=\left(1+\frac{\mathrm{v}}{\mathrm{c}}\right) \overline{\mathrm{H}}^{-}$has not changed.

The radiation pressures on Mirror A and Mirror B do not counterbalance each other anymore and the resulting radiation pressure equals:

$$
\mathbf{P}_{\mathrm{B}}-\mathbf{P}_{\mathrm{A}}=\frac{r^{2}(4 \Delta \mathrm{v}) \mathrm{S}}{\mathrm{c}^{2}}
$$

Equation (10) can be written as [11]:

$$
\mathrm{P}_{\mathrm{B}}-\mathrm{P}_{\mathrm{A}}=\frac{\gamma^{2}(4 \Delta \mathrm{v}) \mathrm{S}}{\mathrm{c}^{2}}=\frac{\gamma^{2}\left(4 \frac{\Delta \mathrm{v}}{\Delta \mathrm{t}}\right) \mathrm{S} \Delta \mathrm{t}}{\mathrm{c}^{2}}=\gamma^{2} \frac{\mathrm{W}}{\mathrm{c}^{2}} \mathrm{a}=\gamma^{2} \mathrm{ma}
$$

In which the acceleration $\mathrm{a}=\frac{\Delta \mathrm{v}}{\Delta \mathrm{t}}$ and the inertia $\mathrm{m}=\frac{\mathrm{W}}{\mathrm{c}^{2}}$. At non-relativistic velocities $\gamma=1$ and (11) turns into the Newton's second law of motion. From (22) also Einstein's famous equation $\mathrm{W}=\mathrm{m} \mathrm{c}^{2}$ follows. In $(11) \mathrm{W}$ is the total confined electromagnetic mass.

Now we can consider electromagnetic confinements without the use of mirrors but electromagnetic confinements through electromagnetic gravitational interaction. By superposition and integration over arbitrary surfaces it is possible to prove that all confined electromagnetic radiation equals (32) and represents electromagnetic mass and follows 
Newton's second law of motion $(\mathrm{F}=\mathrm{m}$ a).

\subsection{The Origin of Gravity:}

It is a misunderstanding that a mass has been attracted by a gravitational field. An interaction force is always an interaction between two fields.

The Electric force is the interaction between two electric fields

The magnetic force is the interaction between two magnetic fields.

The gravity force is the interaction between two gravitational fields.

As an example we choose the propagation of a Laser beam around a black hole in a circular orbit.

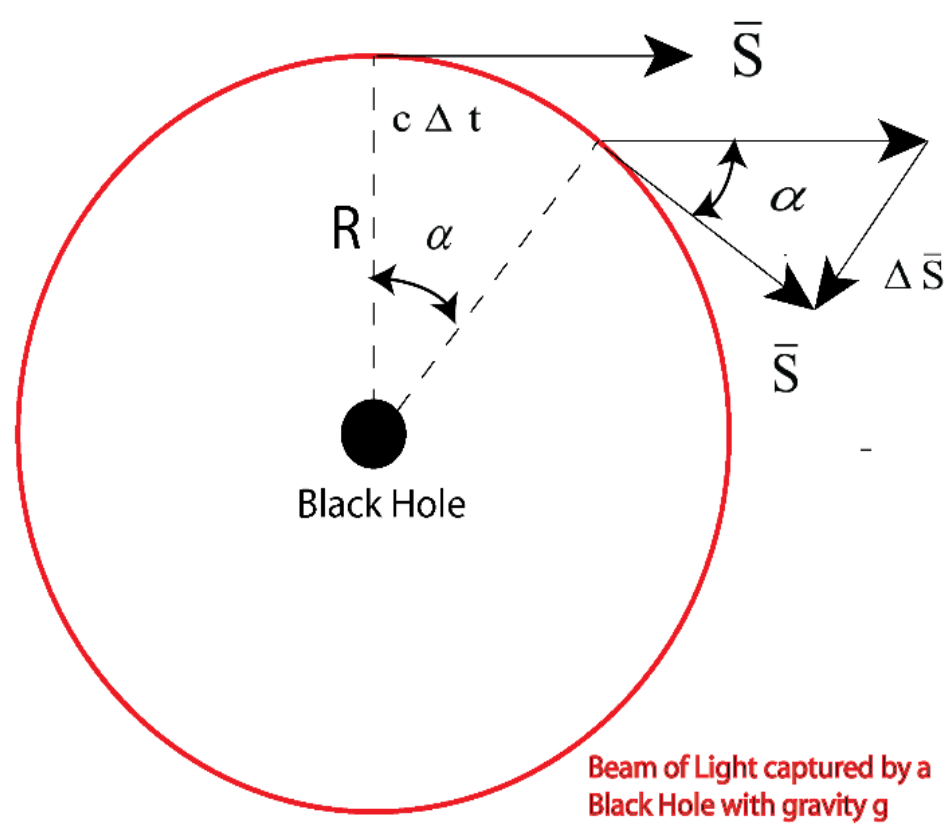

Fig. 12 A LASER beam around a Black Hole captured in a circular orbit around the Black Hole in a Transversal Modus by the Gravitational interaction of the Black Hole with the mass (inertia) of the Laser Beam Light. 
From Figure (5) follows the relationship between de changing in the Poynting vector $\Delta \mathrm{S}$ and the time interval $\Delta \mathrm{t}$ :

$\operatorname{Tan}(\alpha)=\frac{\mathrm{c} \Delta \mathrm{t}}{\mathrm{R}}=\frac{\Delta \mathrm{S}}{\mathrm{S}}-$

$\frac{\Delta \mathrm{S}}{\Delta \mathrm{t}}=\frac{\mathrm{c} \mathrm{S}}{\mathrm{R}}$

Newton's second law:

$\mathrm{F}=\mathrm{m} \cdot \mathrm{a}$

$\mathrm{f}=\left(\frac{\mathrm{F}}{\mathrm{V}}\right)=\left(\frac{\mathrm{m}}{\mathrm{V}}\right) \cdot \mathrm{a}$

$\mathrm{f}=\left(\frac{\mathrm{F}}{\mathrm{V}}\right)=(\rho) \cdot \mathrm{a}$

Equilibrium (interaction) between two gravitational fields $g_{i}$ :

$\mathrm{f}=(\rho) \cdot \mathrm{g}$ (gravitational field $\mathrm{g}_{1}$ of Black Hole

Accelleration of LASER beam: $F=m a=m \frac{d v}{d t}=\frac{d(m v)}{d t}=\frac{d p}{d t}=\frac{1}{c} \frac{d ~ S}{d t}$

$\mathrm{f}=\rho \mathrm{g}=\frac{1}{\mathrm{c}^{2}} \frac{\Delta(\mathrm{S})}{\Delta \mathrm{t}}=\frac{1}{\mathrm{c}^{2}} \frac{\mathrm{c} \mathrm{S}}{\mathrm{R}}=\frac{1}{\mathrm{c}} \frac{\mathrm{S}}{\mathrm{R}}$

The Poynting vector $\overline{\mathrm{S}}$ represents the total energy transport of the electromagnetic radiation per unit surface per unit time $\left[\mathrm{J} / \mathrm{m}^{2} \mathrm{~s}\right]$. Which can be written as the cross product of the Electric Field intensity $\overline{\mathrm{E}}$ and the magnetic Field intensity $\overline{\mathrm{H}}$.

$\overline{\mathrm{S}}=\overline{\mathrm{E}} \times \overline{\mathrm{H}}$

$\mathrm{S}=\mathrm{EH} \operatorname{Sin}\left(90^{\circ}\right)=\mathrm{EH}$

$S=\mathrm{E}^{2} \sqrt{\frac{\varepsilon}{\mu}}$

Substituting equation (10) and (13) in (12) results in an equation for the Event Horizon at radius " $\mathrm{R}$ " of a Transversal Black Hole.

$$
\begin{aligned}
& \rho \mathrm{g}=\frac{1}{\mathrm{c}} \frac{\mathrm{S}}{\mathrm{R}} \\
& \mathrm{R}=\frac{\mathrm{S}}{\rho \mathrm{cg}} \\
& R=\frac{\mathrm{E}^{2} \sqrt{\frac{\varepsilon}{\mu}}}{\rho \mathrm{cg}}=\frac{\mathrm{E}^{2} \sqrt{\frac{\varepsilon}{\mu}}}{\frac{\varepsilon}{\mathrm{c}} \mathrm{E}^{2} \mathrm{~g}} \\
& \mathrm{R}=\frac{\mathrm{c}^{2}}{\mathrm{~g}} \approx \frac{910^{16}}{g}[\mathrm{~m}]
\end{aligned}
$$

Equation (84) represents the perfect equilibrium between the inertia force densities of the 
electromagnetic mass $\frac{1}{c^{2}} \frac{\Delta \overline{\mathrm{S}}}{\Delta \mathrm{t}}$ and the centripetal oriented gravitational force density $\frac{\mathrm{W}}{\mathrm{c}^{2}} \overline{\mathrm{g}}$ acting on the electromagnetic mass. The perfect equilibrium direction where the inertia forces due to the circular orbit of the beam of light are in a perfect balance with the attractive gravitational forces, exists at one defined radius "R" of the beam of light (LASER beam), independent of the intensity of the beam of light and independent of the frequency of the beam of light. Only the acceleration " $\mathrm{g}$ " of the gravitational field determines the radius of equilibrium "R"

$\mathrm{R} \approx \frac{910^{16}}{g}$

In which " $R$ " is the radius of the beam of light and " $g$ " the acceleration of the gravitational field of the "Black Hole".

The Laser beam has been accelerated during the circular orbit. The black hole creates a spherical gravitational field. The gravitational force acting on the LASER beam is the interaction between the gravitational field of the black hole and the gravitational field generated by the accelerated LASER beam. 


\section{References:}

1) Vegt, W. (2021, May 6). Experimental evidence, delivered in "Operational Resource Theory of Imaginarity", proves the physical existence of Sub-Atomic GEONs. https://doi.org/10.31219/osf.io/cqjn9

2) Wim Vegt (2020, October 11). The Illusion of Quantum Mechanical Probability Waves. https://doi.org/10.24018/ejers.2020.5.10.2153

3) Wim Vegt ( 2019, September 29) The Inner Structure of the Photon. https://doi.org/10.24018/ejers.2019.4.9.1535 